\title{
Fuzzy Logic Control System Stability Analysis Based on Lyapunov's Direct Method
}

\author{
R.-E. Precup, M.-L. Tomescu, Şt. Preitl
}

Radu-Emil Precup, Ştefan Preitl

"Politehnica" University of Timişoara

Department of Automation and Applied Informatics

Bd. V. Parvan 2, RO-300223 Timişoara, Romania

E-mail: \{radu.precup, stefan.preitl $\} @$ aut.upt.ro

Marius-Lucian Tomescu

"Aurel Vlaicu" University of Arad

Computer Science Faculty

Complex Universitar M, Str. Elena Dragoi 2, RO-310330 Arad, Romania

E-mail: tom_uav@yahoo.com

\begin{abstract}
A stability analysis method for nonlinear processes controlled by TakagiSugeno (T-S) fuzzy logic controllers (FLCs) is proposed. The stability analysis of these fuzzy logic control systems is done in terms of Lyapunov's direct method. The stability theorem presented here ensures sufficient conditions for the stability of the fuzzy logic control systems. The theorem enables the formulation of a new stability analysis algorithm that offers sufficient stability conditions for nonlinear processes controlled by a class of T-S FLCs. In addition, the paper includes an illustrative example that describes one application of this algorithm in the design of a stable fuzzy logic control system.
\end{abstract}

Keywords: fuzzy logic controller, LaSalle's invariance principle, Lyapunov function candidate.

\section{Introduction}

Fuzzy logic controllers have been proposed for a long time and applied successfully in many applications $[1,2,3,13,14,18]$. A comprehensive work on the proof of stability of fuzzy logic control systems represents one of the challenges in fuzzy control $[6,12,16,17]$. This paper presents a new stability analysis method for fuzzy logic control systems comprising nonlinear processes and T-S FLCs. The advantages of this method with respect to the state-of-the-art result from its specific features. First, it is different to Lyapunov's theorem in several important aspects and allows more applications. In particular, it is well-suited to controlling processes where the derivative of the Lyapunov function candidate is not negative definite. Therefore Lyapunov's direct method can cope with fuzzy control of a wide area of nonlinear dynamic systems. Second, the stability of the closed-loop system is guaranteed by the stability in each active region of the fuzzy rules. So making use of the proposed stability analysis approach determines the inserting of new fuzzy rules become very easy because just the fulfillment of one condition in the stability analysis theorem is needed.

The paper discusses the following topics. Section 2 deals with the description of the accepted class of fuzzy logic control systems. The proposed stability analysis method focused on a stability theorem based on Lyapunov's direct method and the new stability analysis algorithm that guarantees the stability of fuzzy logic control systems are presented in Section 3. Next, Section 4 offers a simple example to validate the theoretical part suggesting ways of applying the proposed algorithm. The conclusions are drawn in Section 5. 


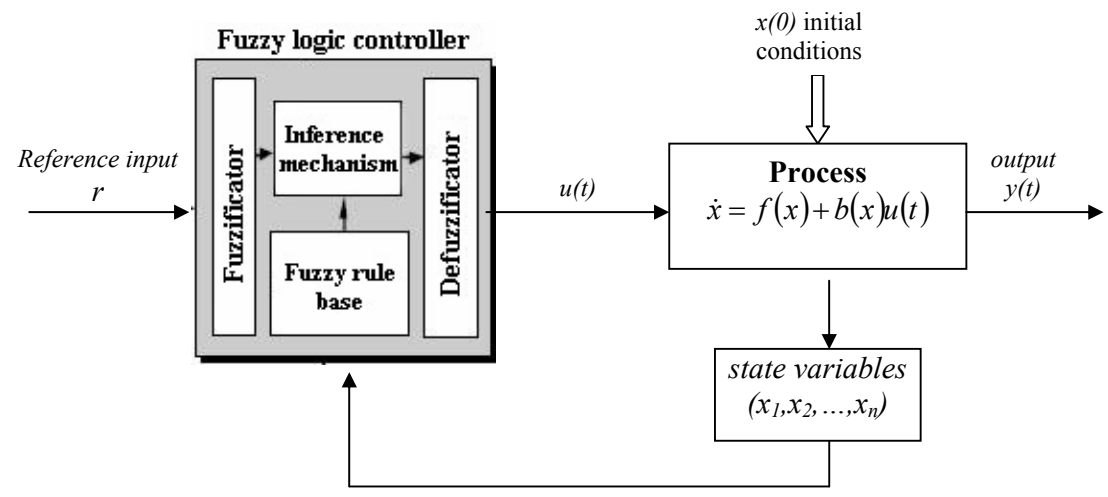

Figure 1: Fuzzy logic control system structure.

\section{Fuzzy Logic Control Systems}

The structure of a fuzzy logic control system consisting of a process controlled by an FLC is presented in Figure 1. Let $X$ be the universe of discourse and consider a single-input $n$-th order nonlinear system of the following form representing the state-space equations of the controlled process:

$$
\dot{x}=f(x)+b(x) u, x\left(t_{0}\right)=x_{0},
$$

where:

$-x \in X, x=\left[x_{1}, x_{2}, \ldots, x_{n}\right]^{T}$ is the state vector;

$-f(x)=\left[f_{1}(x), f_{2}(x), \ldots, f_{n}(x)\right]^{T}, b(x)=\left[b_{1}(x), b_{2}(x), \ldots, b_{n}(x)\right]^{T}$ are functions describing the dynamics of the process, $f, b: D \rightarrow R^{n}$ are locally Lipschitz maps from a domain $D \subset R^{n}$ into $R^{n}$;

$-u$ is the control signal applied to the process input;

- the time variable, $t$, has been omitted to simplify the further formulation;

$-x\left(t_{0}\right)$ is the initial state at time $t_{0}$.

The $i$-th fuzzy (control) rule in the rule base of the T-S FLC base is of the form (2):

$$
\begin{array}{r}
\text { Rule } i \text { : IF } x_{1} \text { IS } X_{i, 1} \text { AND } x_{2} \text { IS } X_{i, 2} \text { AND } \ldots \text { AND } x_{n} \text { IS } X_{i, n} \\
\operatorname{THEN} u=u_{i}(x), i=\overline{1, r}, r \in N^{*},
\end{array}
$$

where $r$ is the total number of rules, $X_{i, 1}, X_{i, 2}, . ., X_{i, n}$ are fuzzy sets that describe the linguistics terms (LTs) of the input variables $x_{k}, k=\overline{1, n}, u=u_{i}(x)$ is the control signal of rule $i$, similar to the case of parallel distributed compensation, and the function AND is a t-norm. $u_{i}$ can be a single value or a function of the state vector, $x$.

The structure presented in Figure $1 \mathrm{cam}$ be viewed as a nonlinear state-feedback control system. However other input variables (to the FLC) can be considered as well instead of the state variables $x_{k}, k=$ $\overline{1, n}$. One simple design of the fuzzy logic control system can be done in terms of parallel distributed compensation.

Each fuzzy rule generates the firing strength defined in (3):

$$
\alpha_{i}(x)=\operatorname{AND}\left(\mu_{i, 1}\left(x_{1}\right), \mu_{i, 2}\left(x_{2}\right) \ldots \mu_{i, n}\left(x_{n}\right)\right) \in[0,1], \forall x \in X, i=\overline{1, r} .
$$


It is assumed that for any $x \in X$ there exists among all rules at least one $\alpha_{i} \in(0,1], i=\overline{1, r}$. The control signal $u$ is a function of $\alpha_{i}$ and $u_{i}$. Applying the weighted sum defuzzification method the output of the FLC is given by

$$
u=\frac{\sum_{i=1}^{r} \alpha_{i} u_{i}}{\sum_{i=1}^{r} \alpha_{i}} .
$$

Definition 1. For any input $x_{0} \in X$ if the firing strength $\alpha_{i}\left(x_{0}\right)$ corresponding to the fuzzy rule $i$ is zero, that fuzzy rule $i, i=\overline{1, r}$, is called an inactive fuzzy rule for the input $x_{0}$; otherwise, it is called an active fuzzy rule.

It should be noted that with $x=x_{0}$ an inactive fuzzy rule will not affect the controller output $u\left(x_{0}\right)$. Hence (4) can be rewritten as follows aiming the consideration of all active fuzzy rules only:

$$
u\left(x_{0}\right)=\frac{\sum_{i=1, \alpha_{i} \neq 0}^{r} \alpha_{i}\left(x_{0}\right) u_{i}\left(x_{0}\right)}{\sum_{i=1, \alpha_{i} \neq 0}^{r} \alpha_{i}\left(x_{0}\right)} .
$$

Definition 2. An active region of the fuzzy rule $i$ is defined as a set

$$
X_{i}^{A}=\left\{x \in X \mid \alpha_{i}(x) \neq 0\right\}, i=\overline{1, r} .
$$

\section{Stability Analysis}

The stability analysis presented in this paper is based on LaSalle's invariance principle cited and analyzed in [11]. This Section is concentrated on the formulation and proof of Theorem 1 that ensures sufficient conditions for the stability of nonlinear processes controlled by T-S FLCs.

The Lyapunov function candidate $V: R^{n} \rightarrow R, V(x)=x^{T} P x$ is considered. It is positive and unbounded, where $P \in R^{n \times n}$ is a positive definite matrix. Considering the state trajectories fulfilling (1) in order to obtain the closed-loop system dynamics, it results that $V$ has continuous partial derivatives and the derivatives of $V$ with respect to time expressed in terms of (7):

$$
\begin{aligned}
& \dot{V}(x)=\dot{x}^{T} P x+x^{T} P \dot{x}=(f(x)+b(x) u(x))^{T} P x+ \\
& +x^{T} P(f(x)+b(x) u(x))=F(x)+B(x) u(x),
\end{aligned}
$$

where:

$$
F(x)=f(x)^{T} P x+x^{T} P f(x), B(x)=b(x)^{T} P x+x^{T} P b(x) .
$$

The following sets are defined to be used in the stability analysis:

$$
B^{0}=\{x \in X \mid B(x)=0\}, B^{+}=\{x \in X \mid B(x)>0\}, B^{-}=\{x \in X \mid B(x)<0\} .
$$

The main result is given by the following Theorem.

Theorem 3. Let the process be described by (1) with $x=0 \in R^{n}$ an equilibrium point. If there exists a function $V: R^{n} \rightarrow R, V(x)=x^{T} P x, P \in R^{n \times n}$, positive definite, unbounded and fulfilling 1, 2 and 3:

1. $F(x) \leq 0, \forall x \in B^{0}$,

2. $u_{i}(x) \leq-\frac{F(x)}{B(x)}$ for $x \in X_{i}^{A} \cap B^{+}$and $u_{i}(x) \geq-\frac{F(x)}{B(x)}$ for $x \in X_{i}^{A} \cap B^{-}, i=\overline{1, r}$, 
3. the set $\{x \in X \mid \dot{V}(x)=0\}$ contains no state trajectories except the trivial one, $x(t)=0$ for $t \geq 0$,

then the closed-loop system composed by the T-S FLC and the process (1) will be globally asymptotically stable in the sense of Lyapunov at the origin.

Proof. By the definition of $V$ it results that $V(0)=0, V(x)>0, \forall x \neq 0$ and $V(x)=x^{T} P x \rightarrow \infty$ as $\|x\| \rightarrow \infty$. Further on, it will be proved that $\dot{V}$ is negative semi-definite with respect to time employing (7). An arbitrary initial state vector $x_{0} \in X$ is accepted. Then the following three cases are possible.

Case 1: $B\left(x_{0}\right)$ is strictly positive. From the condition 2 of Theorem 1 it results that:

$$
\begin{gathered}
u_{i}\left(x_{0}\right) \leq-\frac{F\left(x_{0}\right)}{B\left(x_{0}\right)} \Rightarrow u\left(x_{0}\right)=\frac{\sum_{i=1, \alpha_{i} \neq 0}^{r} \alpha_{i}\left(x_{0}\right) u_{i}\left(x_{0}\right)}{\sum_{i=1, \alpha_{i} \neq 0}^{r} \alpha_{i}\left(x_{0}\right)} \leq \frac{-\frac{F\left(x_{0}\right)}{B\left(x_{0}\right)} \sum_{i=1, \alpha_{i} \neq 0}^{r} \alpha_{i}\left(x_{0}\right)}{\sum_{i=1, \alpha_{i} \neq 0}^{r} \alpha_{i}\left(x_{0}\right)}=-\frac{F\left(x_{0}\right)}{B\left(x_{0}\right)} \Rightarrow \\
\Rightarrow \dot{V}\left(x_{0}\right)=F\left(x_{0}\right)+B\left(x_{0}\right) u\left(x_{0}\right) \leq F\left(x_{0}\right)+B\left(x_{0}\right)\left(-\frac{F\left(x_{0}\right)}{B\left(x_{0}\right)}\right)=0 .
\end{gathered}
$$

Therefore,

$$
u_{i}\left(x_{0}\right) \leq-\frac{F\left(x_{0}\right)}{B\left(x_{0}\right)} \Rightarrow \dot{V}\left(x_{0}\right) \leq 0 .
$$

Case 2: $B\left(x_{0}\right)$ is strictly negative. Once more, from the condition 2 of Theorem 1 it results that

$$
\begin{gathered}
u_{i}\left(x_{0}\right) \geq-\frac{F\left(x_{0}\right)}{B\left(x_{0}\right)} \Rightarrow u\left(x_{0}\right)=\frac{\sum_{i=1, \alpha_{i} \neq 0}^{r} \alpha_{i}\left(x_{0}\right) u_{i}\left(x_{0}\right)}{\sum_{i=1, \alpha_{i} \neq 0}^{r} \alpha_{i}\left(x_{0}\right)} \geq \frac{-\frac{F\left(x_{0}\right)}{B\left(x_{0}\right)} \cdot \sum_{i=1, \alpha_{i} \neq 0}^{r} \alpha_{i}\left(x_{0}\right)}{\sum_{i=1, \alpha_{i} \neq 0}^{r} \alpha_{i}\left(x_{0}\right)}=-\frac{F\left(x_{0}\right)}{B\left(x_{0}\right)} \Rightarrow \\
\Rightarrow \dot{V}\left(x_{0}\right)=F\left(x_{0}\right)+B\left(x_{0}\right) u\left(x_{0}\right) \leq F\left(x_{0}\right)+B\left(x_{0}\right)\left(-\frac{F\left(x_{0}\right)}{B\left(x_{0}\right)}\right)=0 .
\end{gathered}
$$

Therefore,

$$
u_{i}\left(x_{0}\right) \geq-\frac{F\left(x_{0}\right)}{B\left(x_{0}\right)} \Rightarrow \dot{V}\left(x_{0}\right) \leq 0 .
$$

Case 3: $x_{0} \in B^{\circ}$. In this case using the condition 1 in Theorem 1 the result will be $F\left(x_{0}\right) \leq 0$. Hence,

$$
\dot{V}\left(x_{0}\right)=F\left(x_{0}\right)+B\left(x_{0}\right) u\left(x_{0}\right)=F\left(x_{0}\right) \leq 0 .
$$

From the above three cases it is obtained that

$$
\dot{V}(x) \leq 0, \forall x \in X .
$$

In conclusion, the derivative with respect to time of the Lyapunov function candidate, $\dot{V}$, is negative semi-definite.

The condition 3 ensures the fulfilment of LaSalle's invariance principle. This justifies the fact that the equilibrium point at the origin is globally asymptotically stable.

The proof is now complete.

The stability theorem presented here ensures sufficient conditions for the stability of the fuzzy logic control system described in Section 2. So it has been proved that if the Lyapunov function candidate is negative semi-definite in the active region of each fuzzy rule then, the closed-loop system will be globally asymptotically stable in the sense of Lyapunov. 
The conditions 1 and 2 in Theorem 1 guarantee that the function $\dot{V}$ is negative semi-definite in the active region of each fuzzy rule. The condition 3 proves that the set $\{0\}$ is the largest invariance set in $\{x \in X \mid \dot{V}(x)=0\}$. By LaSalle's invariance principle it has been guaranteed that the fuzzy logic control system, comprising the nonlinear process described by (1) and the T-S FLC, is globally asymptotically stable in the sense of Lyapunov at the origin.

The stability analysis algorithm ensuring the stability of the class of fuzzy logic control systems considered in Section 2 is based on Theorem 1. It consists of the following steps:

1. Set the Lyapunov function candidate $V$ (i.e. set $P$ ).

2. Check that the set $\{x \in X \mid \dot{V}(x)=0\}$ contains no state trajectories except the trivial one, $x(t)=0$ for $t \geq 0$.

3. Determine $F(x), B(x), B^{\circ}, B^{-}, B^{+}$.

4. If $F(x) \leq 0, \forall x \in B^{0}$ then go to step 5. Else go to step 1 .

5. For each fuzzy control rule $i$ determine $u_{i}$ such that $u_{i}(x) \leq-\frac{F(x)}{B(x)}$ for $x \in X_{i}^{A} \cap B^{+}$and $u_{i} \geq-\frac{F(x)}{B(x)}$ for $x \in X_{i}^{A} \cap B^{-}, i=\overline{1, r}$.

The application of this algorithm will be illustrated in the next Section.

\section{Illustrative example}

This Section is dedicated to the validation of the theoretical results derived in Section 3 by the design of a stable fuzzy logic control system with T-S FLC controlling a nonlinear process, the inverted pendulum on a cart system. This simple mechanical system is representative to model a class of attitude control problems whose goal is to maintain permanently the desired vertically oriented position. Since the inverted pendulum is a nonlinear system, the basic balance equations for the system are derived firstly and put into the standard state-space form. Given an inverted pendulum mounted on a cart as shown in Figure 2, the first principle nonlinear equations are applied in the sequel. Assuming that the rod is massless and that the cart mass and the point mass at the upper end of the inverted pendulum are denoted as $M$ and $m$, respectively, there is an externally $x$-directed force on the cart, $F(t)$, and the gravity force acts on the point mass at all times. The coordinate system is defined according to Figure 2, where $x(t)$ represents the cart position and $\theta(t)$ is the tilt angle referenced to the vertical upward direction.

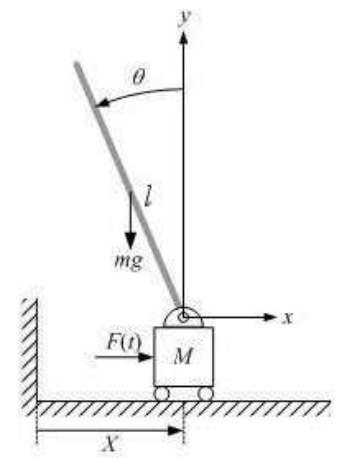

Figure 2: Variables related to the inverted pendulum on a cart system.

The differential equation that describes the behavior of the simplified system, playing the role of controlled process, is usually written as

$$
(m+M) \cdot l^{2} \cdot \ddot{\theta}-(m+M) \cdot l \cdot g \cdot \sin (\theta)=-u,
$$


where:

$M$ - the mass of the cart,

$m$ - the mass of the pendulum,

$l$ - the length of pendulum (distance to the center of mass),

$x$ - the cart position coordinate,

$\theta$ - the pendulum angle with respect to the vertical position,

$u$ - the control signal, equal to the externally $x$-directed force, $u=F$.

The state vector consists of the angle, $\theta$, and the angular velocity of the pendulum, $\dot{\theta}$. Therefore, the two state variables are defined as $z_{1}$ and $z_{2}$, where $z_{1} \in[-80,80], z_{2} \in[-30,30], z_{1}(t)=\theta(t)$ and $z_{2}(t)=\dot{\theta}(t)$. In order to write equation (19) in terms of state variables, they are substituted resulting in

$$
\dot{z}=f(z)+b(z) u,
$$

where: $z=\left[\begin{array}{c}z_{1} \\ z_{2}\end{array}\right]$ - state vector, $f(z)=\left[\begin{array}{c}z_{2} \\ \frac{g}{l} \sin \left(z_{1}\right)\end{array}\right], b(z)=\left[\begin{array}{c}0 \\ -\frac{1}{(m+M) l^{2}}\end{array}\right]$.

The goal of fuzzy logic control system design, to be presented as follows, is to ensure the upright stabilization of the pendulum aiming the setpoint value of $z, z=0$. The design starts with setting the fuzzification module of the T-S FLC. Figures 3 and 4 illustrates the membership functions corresponding to the LTs of the linguistic variables $z_{1}$ and $z_{2}$. The three LTs representing Positive, Zero and Negative values are noted by $\mathrm{P}, \mathrm{Z}$ and $\mathrm{N}$, respectively.

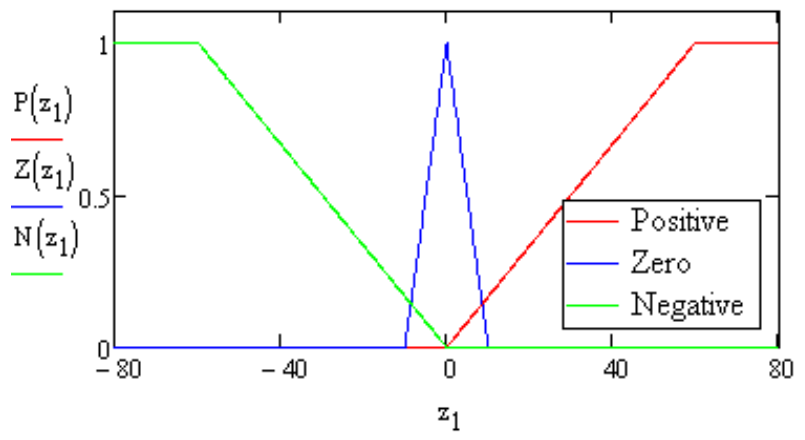

Figure 3: Membership functions of $z_{1}$.

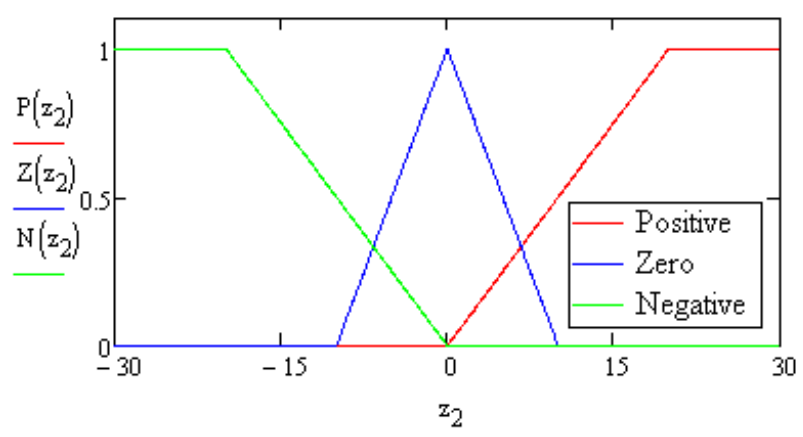

Figure 4: Membership functions of $z_{2}$. 
The inference engine of the FLC employs the MIN and MAX operators and it is assisted by the complete rule base illustrated in Table 1. The weighted sum defuzzification method is used in the T-S FLC structure. Summarizing, the only parameters to be calculated are the consequents $u_{i}$ in the 9 fuzzy control rules.

Table 1

Fuzzy Control Rule Base

\begin{tabular}{cccc}
\hline Rule & \multicolumn{2}{c}{ Antecedent } & Consequent \\
\hline & $z_{1}$ & $z_{2}$ & $u$ \\
\hline 1 & $\mathrm{P}$ & $\mathrm{P}$ & $u_{1}$ \\
2 & $\mathrm{~N}$ & $\mathrm{~N}$ & $u_{2}$ \\
3 & $\mathrm{P}$ & $\mathrm{N}$ & $u_{3}$ \\
4 & $\mathrm{~N}$ & $\mathrm{P}$ & $u_{4}$ \\
5 & $\mathrm{P}$ & $\mathrm{Z}$ & $u_{5}$ \\
6 & $\mathrm{~N}$ & $\mathrm{Z}$ & $u_{6}$ \\
7 & $\mathrm{Z}$ & $\mathrm{P}$ & $u_{7}$ \\
8 & $\mathrm{Z}$ & $\mathrm{N}$ & $u_{8}$ \\
9 & $\mathrm{Z}$ & $\mathrm{Z}$ & $u_{9}$
\end{tabular}

The algorithm presented in Section 3 will be applied as follows in order to find the values of $u_{i}$ for which the system (19) can be stabilized by the above described T-S FLC.

Step 1: The Lyapunov function candidate $V(z)=z^{T} P z=z_{1}^{2}+z_{2}^{2}$ is considered, where $P=\left(\begin{array}{cc}1 & 0 \\ 0 & 1\end{array}\right)$. Therefore $V$ is positive. If $\|z\| \rightarrow \infty$ then $V(z) \rightarrow \infty$. $V(z)>0, \forall z \neq 0$.

Step 2: The derivative is $\dot{V}(z)=2 z_{2}\left(z_{1}+\frac{g}{l} \sin \left(z_{1}\right)-\frac{1}{(m+M) l^{2}} u\right)$ and $\dot{V}(0)=0$. Assume that there is a trajectory with $z_{2}(t)=0$ and $z_{1}(t) \neq 0$. Then $\frac{d}{d t} z_{2}(t)=\frac{g}{l} \sin \left(z_{1}(t)\right)-\frac{1}{(m+M) l^{2}} u(t) \neq 0$, which means that $z_{2}(t)$ can not stay constant. Hence, $z(t)=0$ is the only possible state trajectory for which $\dot{V}(z)=0$. So the set $\{z \in X \mid \dot{V}(z)=0\}$ contains no trajectory of the system except the trivial trajectory $z(t)=0$ for $t \geq 0$.

Step 3: The expressions of $F$ and $B$ are:

$$
F(z)=2 z_{2}\left(z_{1}+\frac{g}{l} \sin \left(z_{1}\right)\right), B(z)=-\frac{2 z_{2}}{(m+M) l^{2}} .
$$

The following elements necessary in Theorem 1 obtain the particular values expressed in (23) to (26):

$$
\begin{gathered}
B^{\mathrm{o}=}=\left\{\left(z_{1}, 0\right) \in X \mid z_{1} \in[-1,1]\right\}, B^{+}=\left\{\left(z_{1}, z_{2}\right) \in X \mid z_{2}<0\right\}, B^{-}=\left\{\left(z_{1}, z_{2}\right) \in X \mid z_{2}>0\right\}, \\
-\frac{F(z)}{B(z)}=l(m+M)\left(z_{1} l+g \sin \left(z_{1}\right)\right) .
\end{gathered}
$$

Step 4: If $z \in B^{\circ}$ then $z_{2}=0$ and $F(z)=0$.

Step 5: Each rule will be analyzed further on. This is not a complex task since only 9 rules are involved. For rule 1: $z_{1}$ IS P, $z_{2}$ IS P. So $X_{1}^{A}=(0,80] \times(0,30], X_{1}^{A} \cap B^{+}=\emptyset$ and $X_{1}^{A} \cap B^{-}=(0,80] \times(0,30]$. Thus, $u_{1}(z) \geq-\frac{F(z)}{B(z)}=l(m+M)\left(z_{1} l+g \sin \left(z_{1}\right)\right)$. It is taken $u_{1}(z)=l(m+M)\left(z_{1} l+g\right)$, and this function fulfills the condition 2 in Theorem 1.

For rule $2: z_{1}$ IS N, $z_{2}$ IS N. So $X_{2}^{A}=[-80,0) \times[-30,0), X_{2}^{A} \cap B^{+}=[-80,0) \times[-30,0)$ and $X_{2}^{A} \cap$ $B^{-}=\emptyset$. Thus $u_{2} \leq-\frac{F(z)}{B(z)}=l(m+M)\left(z_{1} l+g \sin \left(z_{1}\right)\right)$. It is taken $u_{2}(z)=l(m+M)\left(z_{1} l-g\right)$, and this function fulfills the condition 2 in Theorem 1. 
For rule 3: $z_{1}$ IS P, $z_{2}$ IS N. So $X_{3}^{A}=(\mathrm{o}, 8 \mathrm{o}] \times(-30,0], X_{3}^{A} \cap B^{-}=\emptyset$ and $X_{3}^{A} \cap B^{+}=(\mathrm{o}, 8 \mathrm{o}] \times(-30,0]$. Thus $u_{3}(z) \leq-\frac{F(z)}{B(z)}=l(m+M)\left(z_{1} l+g \sin \left(z_{1}\right)\right)$. It is taken $u_{3}(z)=-z_{1}$, and this function fulfills again the given condition.

For rule 4: $z_{1}$ IS N, $z_{2}$ IS P. So $X_{4}^{A}=[-80,0) \times(0,30], X_{4}^{A} \cap B^{+}=\emptyset$ and $X_{4}^{A} \cap B^{-}=[-80,0) \times(0,30]$. Thus $u_{4}(z) \geq-\frac{F(z)}{B(z)}=l(m+M)\left(z_{1} l+g \sin \left(z_{1}\right)\right)$. It is taken $u_{4}(z)=-z_{1}$, and this function fulfills the given condition.

For rule 5: $z_{1}$ IS P, $z_{2}$ IS Z. So $X_{5}^{A}=(0,80] \times(-5,5)$. Thus, two possible cases will occur:

a) for $z \in X_{5}^{A} \cap B^{-}=(0,80] \times[0,5) \Rightarrow u_{5}(z) \geq-\frac{F(z)}{B(z)}=l(m+M)\left(z_{1} l+g \sin \left(z_{1}\right)\right)$ and

b) for $z \in X_{5}^{A} \cap B^{+}=(0,80] \times(-5,0] \Rightarrow u_{5}(z) \leq-\frac{F(z)}{B(z)}=l(m+M)\left(z_{1} l+g \sin \left(z_{1}\right)\right)$.

In order to satisfy both conditions it is chosen $u_{5}(z)=l(m+M)\left(z_{1} l+g \sin \left(z_{1}\right)\right)$.

For rule 6: $z_{1}$ IS N, $z_{2}$ IS Z. So $X_{6}^{A}=[-80,0) \times(-5,5)$. The result will be:

a) for $z \in X_{6}^{A} \cap B^{-}=[-80,0) \times(0,5) \Rightarrow u_{6}(z) \geq-\frac{F(z)}{B(z)}=l(m+M)\left(z_{1} l+g \sin \left(z_{1}\right)\right)$ and

b) for $z \in X_{6}^{A} \cap B^{+}=[-80,0) \times(-5,0) \Rightarrow u_{6}(z) \leq-\frac{F(z)}{B(z)}=l(m+M)\left(z_{1} l+g \sin \left(z_{1}\right)\right)$.

In order to satisfy both conditions it is chosen $u_{6}(z)=l(m+M)\left(z_{1} l+g \sin \left(z_{1}\right)\right)$.

For rule 7: $z_{1}$ IS Z, $z_{2}$ IS P. So $X_{7}^{A}=(-10,10) \times(0,30], X_{7}^{A} \cap B^{+}=\emptyset$ and $X_{7}^{A} \cap B^{-}=(-10,10) \times$ $(0,30]$. Thus $u_{7}(z) \geq-\frac{F(z)}{B(z)}=l(m+M)\left(z_{1} l+g \sin \left(z_{1}\right)\right)$. It is set $u_{7}(z)=l(m+M)\left(z_{1} l+g\right)$ to fulfill the condition 2 in Theorem 1.

For rule 8: $z_{1}$ IS Z, $z_{2}$ IS N. So $X_{8}^{A}=(-10,10) \times[-30,0), X_{8}^{A} \cap B^{+}=(-10,10) \times[-30,0)$ and $X_{8}^{A} \cap B^{-}=\emptyset$. Thus $u_{8}(z) \leq-\frac{F(z)}{B(z)}=l(m+M)\left(z_{1} l+g \sin \left(z_{1}\right)\right)$. It is set $u_{8}(z)=l(m+M)\left(z_{1} l-g\right)$ to fulfill the condition 2 in Theorem 1.

For rule 9: $z_{1}$ IS Z, $z_{2}$ IS Z. So $X_{9}^{A}=(-10,10) \times(-5,5)$. This will yield:

a) for $z \in X_{9}^{A} \cap B^{+}=(-10,10) \times(-5,0) \Rightarrow u_{9}(z) \leq-\frac{F(z)}{B(z)}=l(m+M)\left(z_{1} l+g \sin \left(z_{1}\right)\right)$ and

b) for $z \in X_{9}^{A} \cap B^{-}=(-10,10) \times(0,5) \Rightarrow u_{9}(z) \geq-\frac{F(z)}{B(z)}=l(m+M)\left(z_{1} l+g \sin \left(z_{1}\right)\right)$.

In order to satisfy both conditions it is set $u_{9}(z)=l(m+M)\left(z_{1} l+g \sin \left(z_{1}\right)\right)$.

Concluding, from Theorem 1 it results that the closed-loop system composed by the nonlinear process modeled in (19) and the T-S FLC designed here is globally asymptotically stable in the sense of Lyapunov at the origin. Considering the values of process parameters $m=0.5, M=0.5, l=1, g=9.8$, the responses of $z_{1}$ and $z_{2}$ versus time in the closed-loop system are presented in Figures 5 to 8 for different initial conditions.

\section{Summary and Conclusions}

A new approach to the global asymptotic stability analysis of fuzzy logic control systems employing T-S FLCs dedicated to a class of nonlinear processes has been introduced. The example proves how the stability analysis algorithm suggested here can be applied to the design of a stable fuzzy logic control system for a nonlinear process. The new stability approach can be applied also in situations when the system has an equilibrium point different to the origin and / or the setpoint is nonzero by an appropriately defined state transformation [15]. 


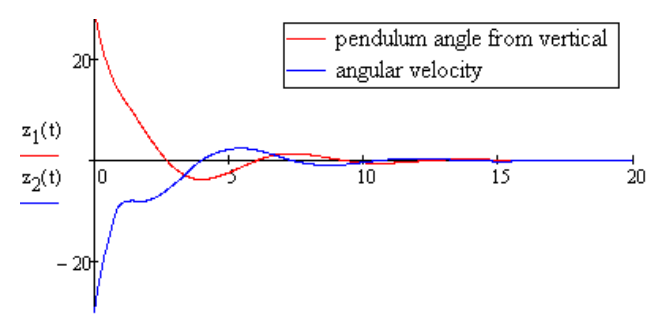

$t[s]$

Figure 5: State variables versus time for fuzzy logic control system with T-S FLC in the condition $z_{1}(0)=30$ and $z_{2}(0)=-30$.

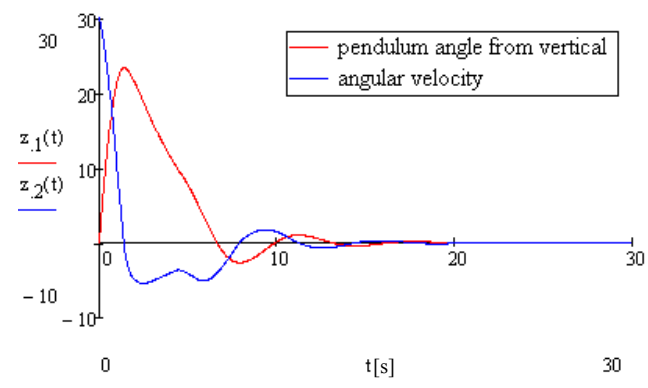

Figure 6: State variables versus time for fuzzy logic control system with T-S FLC in the condition $z_{1}(\mathrm{o})=0$ and $z_{2}(\mathrm{o})=30$.

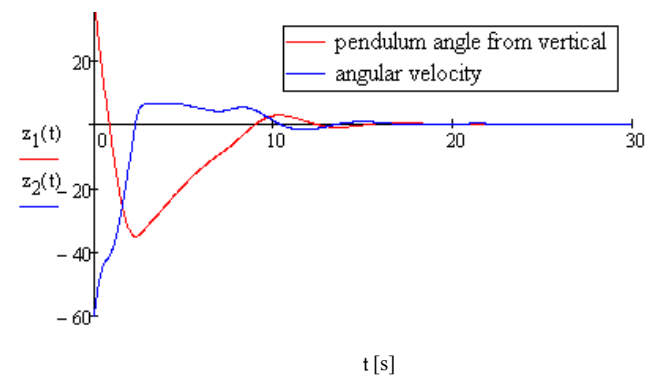

Figure 7: State variables versus time for fuzzy logic control system with T-S FLC in the condition $z_{1}(\mathrm{o})=4 \mathrm{o}$ and $z_{2}(\mathrm{o})=-6 \mathrm{o}$.

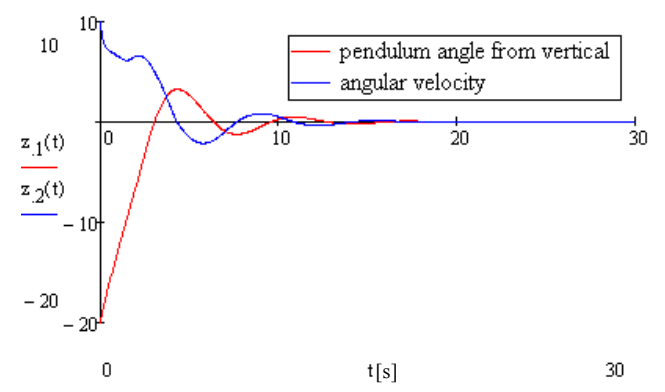

Figure 8: State variables versus time for fuzzy logic control system with T-S FLC in the condition $z_{1}(0)=-20$ and $z_{2}(0)=10$.

The stability analysis algorithm suggested in this paper canbe applied also when the rule base (2) of the T-S FLC is not complete. However interpolation techniques $[10,19]$ are needed in the imple- 
mentation of the T-S FLC. They require the re-assessment of the stability conditoins derived prior to the implementation.

Further research will be concentrated on new applications of the proposed algorithm to several classes of processes $[4,5,7,8,9,17,20]$. The complex applications require the computer-aided design of the Takagi-Sugeno fuzzy logic controllers employing the stability analysis algorithm proposed in this paper to strive for increased generality.

\section{Acknowledgement}

The support from the CNCSIS and CNMP of Romania is acknowledged.

\section{Bibliography}

[1] M. M. Bălaş, V. E. Bălaş, World Knowledge for Control Applications by Fuzzy-Interpolative Systems, International Journal of Computers, Communications \& Control, Vol. III, Suppl. Issue: Proceedings of ICCCC 2008, pp. 28-32, 2008.

[2] A. Bazoula, M.S. Djouadi, H. Maaref, Formation Control of Multi-Robots via Fuzzy Logic Technique, International Journal of Computers, Communications \& Control, Vol. III, Suppl. Issue: Proceedings of ICCCC 2008, pp. 179-184. 2008.

[3] D. Bellomo, D. Naso, and R. Babuška, Adaptive fuzzy control of a non-linear servo-drive: theory and experimental results, Engineering Applications of Artificial Intelligence, Vol. 21, Issue 6, pp. 846-857, 2008.

[4] S. Blažič and I. Škrjanc, Design and stability analysis of fuzzy model-based predictive control - a case study, Journal of Intelligent and Robotic Systems, Vol. 49, Issue 3, pp. 279-292, 2007.

[5] S. Ertugrul, Predictive modeling of human operators using parametric and neuro-fuzzy models by means of computer-based identification experiment, Engineering Applications of Artificial Intelligence, Vol. 21, Issue 2, pp. 259-268, 2008.

[6] G. Feng, A survey on analysis and design of model-based fuzzy control systems, IEEE Transactions on Fuzzy Systems, Vol. 14, Issue 5, pp. 676-697, 2006.

[7] D. Hladek, J. Vascak, and P. Sincak, Multi-robot control system for pursuit-evasion problem, Journal of Electrical Engineering, Vol. 60, Issue 3, pp. 143-148, 2009.

[8] L. Horváth and I. J. Rudas, Towards content oriented integration of product and robot system models, International Symposium on Logistics and Industrial Informatics, Wildau, pp. 7-12, 2007.

[9] G.-H. Hwang, D.-W. Kim, J.-H. Lee, and Y.-J. An, Design of fuzzy power system stabilizer using adaptive evolutionary algorithm, Engineering Applications of Artificial Intelligence, Vol. 21, Issue 1, pp. 86-96, 2008.

[10] Z. C. Johanyák and S. Kovács: Sparse fuzzy system generation by rule base extension, 11th IEEE International Conference of Intelligent Engineering Systems, Budapest, pp. 99-104, 2007.

[11] H. K. Khalil, Nonlinear Systems, Third Edition, Prentice Hall, Englewood Cliffs, NJ, 2002.

[12] K. Michels, F. Klawonn, R. Kruse, and A. Nürnberger, Fuzzy Control: Fundamentals, Stability and Design of Fuzzy Controllers, Springer Verlag, Berlin, Heidelberg, New York, 2006. 
[13] B. M. Mohan and A. Sinha, Analytical structure and stability analysis of a fuzzy PID controller, Applied Soft Computing, Vol. 8, Issue 1, pp. 749-758, 2008.

[14] A. G. Perry, G. Feng, Y.-F. Liu, and P. C. Sen, A design method for PI-like fuzzy logic controllers for DC-DC converter, IEEE Transactions on Industrial Electronics, Vol. 54, Issue 5, pp. 2688-2696, 2007.

[15] R.-E. Precup, S. Preitl, and G. Faur, PI predictive fuzzy controllers for electrical drive speed control: methods and software for stable development, Computers in Industry, Vol. 52, Issue 3, pp. 253-270, 2003.

[16] R.-E. Precup, S. Preitl, I. J. Rudas, M. L. Tomescu, and J. K. Tar, Design and experiments for a class of fuzzy controlled servo systems, IEEE/ASME Transactions on Mechatronics, Vol. 13, Issue 1, pp. 22-35, 2008.

[17] E. Tian and C. Peng, Delay-dependent stability analysis and synthesis of uncertain T-S fuzzy systems with time-varying delay, Fuzzy Sets and Systems, Vol. 157, Issue 4, pp. 544-559, 2006.

[18] T. Vesselenyi, S. Dziţac, I. Dziţac and M.-J. Manolescu, Fuzzy and Neural Controllers for a Pneumatic Actuator, International Journal of Computers, Communications \& Control, Vol. II, No. 4, pp. 375-387, 2007.

[19] K. W. Wong, D. Tikk, T. D. Gedeon, and L. T. Kóczy, Fuzzy rule interpolation for multidimensional input spaces with applications: a case study, IEEE Transactions on Fuzzy Systems, Vol. 13, Issue 6, pp. 809-819, 2005.

[20] J. Yoneyama, Robust stability and stabilization for uncertain Takagi-Sugeno fuzzy time-delay systems, Fuzzy Sets and Systems, Vol. 158, Issue 2, pp. 115-134, 2007.

Radu-Emil Precup was born in Lugoj, Romania, in 1963. He received the Dipl.Ing. degree (Hons.) in Automation and Computers from the "Traian Vuia" Polytechnic Institute of Timişoara, Romania, in 1987, the diploma in Mathematics from the West University of Timişoara in 1993, and the $\mathrm{PhD}$ degree in Automatic Systems from the "Politehnica" University of Timisoara (PUT), Romania, in 1996. From 1987 to 1991, he was with Infoservice S.A., Timişoara. He is currently with the PUT, where he became a Professor in the Department of Automation and Applied Informatics, in 2000, and he is currently a Doctoral Supervisor of Automation and Systems Engineering. He is also an Honorary Professor with the Budapest Tech Polytechnical Institution, Budapest, Hungary. He is the author or coauthor of more than 120 papers published in various scientific journals, refereed conference proceedings, and contributions to books.

Marius L. Tomescu was born in Arad, Romania, in 1967. He received his MSc degree in Computer Science from the West University of Timişoara, Romania, in 1993, and the $\mathrm{PhD}$ degree in Automation from the "Politehnica" University of Timişoara, Romania, in 2008. From 1993 to 2008, he was with Grup Service S.R.L., Arad. He is with the "Aurel Vlaicu" University of Arad, where he has been a Lecturer in the Department of Computer Science since 2002. His research interests include fuzzy control applications and stability analysis of fuzzy control systems. He is the author or coauthor of more than 30 papers published in various scientific journals and refereed conference proceedings. Dr. Tomescu is a member of the Romanian Society of Control Engineering and Technical Informatics. 
Ştefan Preitl was born in Timişoara, Romania, in 1943. He received the Dipl.Ing. degree in Electrical Engineering, and the PhD degree in Measurement Techniques from the "Traian Vuia" Polytechnic Institute of Timişoara in 1966 and 1983, respectively. From 1967 to 1972, he was with Electromotor S.A., Timişoara. He is currently with the "Politehnica" University of Timişoara, Timişoara, where he became a Professor in the Department of Automation and Applied Informatics, in 1992, and he is currently a Doctoral Supervisor of Automation and Systems Engineering. He is also an Honorary Professor with the Budapest Tech Polytechnical Institution, Budapest, Hungary. He is the author or coauthor of more than 170 papers published in various scientific journals, refereed conference proceedings, and books in the field of automatic control. 